\title{
MS-275 sensitizes osteosarcoma cells to Fas ligand- induced cell death by increasing the localization of Fas in membrane lipid rafts
}

\author{
K Rao-Bindal ${ }^{1}$, Z Zhou ${ }^{1}$ and ES Kleinerman ${ }^{\star, 1}$
}

Fas expression is inversely correlated with the metastatic potential of osteosarcoma (OS) cells to the lungs. Fas ${ }^{+}$cells are rapidly eliminated when they enter the lungs via their interaction with constitutive Fas ligand (FasL) on the lung epithelium, whereas Fas ${ }^{-}$OS cells escape this FasL-induced apoptosis and survive in the lung microenvironment. Upregulation of Fas expression in established OS lung metastases results in tumor regression. Here, we demonstrate that treatment of Fas OS cells with the histone deacetylase inhibitor MS-275 results in the upregulation of Fas mRNA and sensitizes these cells to FasL-induced apoptosis. However, flow cytometry analysis revealed that Fas cell surface protein expression was not significantly increased. Rather, we observed increased levels of Fas within the membrane lipid rafts, as demonstrated by an increase in Fas expression in detergent-insoluble lipid raft fractions and colocalization with GM1+ lipid rafts. We had previously shown that MS-275 treatment inhibited expression of the anti-apoptotic cellular FLICE-inhibitory protein (c-FLIP). Here, we demonstrated that transfection of cells with short hairpin RNA to c-FLIP also resulted in the localization of Fas to lipid rafts. Overall, our studies indicate that MS-275 sensitizes OS cells to FasL by upregulating the expression of Fas in membrane lipid rafts, which correlates with the C-FLIP-dependent distribution of Fas to lipid rafts.

Cell Death and Disease (2012) 3, e369; doi:10.1038/cddis.2012.101; published online 9 August 2012

Subject Category: Cancer

Fas and Fas ligand (FasL) are complementary receptor-ligand proteins. ${ }^{1}$ Fas is expressed in many types of tissues, whereas FasL is expressed in only four organs in the body, one of which is the lungs. ${ }^{1,2}$ We previously demonstrated that Fas expression is inversely correlated with metastatic potential of osteosarcoma (OS) cells. ${ }^{3}$ This finding is highly significant because the $\mathrm{FasL}^{+}$lung is the most common, and usually the only, site for OS metastasis. ${ }^{4}$ We have also shown that agents that upregulate Fas expression induce the regression of established OS lung metastasis in vivo. ${ }^{5-8}$ This therapeutic activity depends on an intact Fas signaling pathway. ${ }^{7}$ Therefore, understanding the mechanism of how drugs induce Fas upregulation or enhance sensitivity to FasL may help identify novel therapeutic agents for the treatment of patients with OS lung metastases. For therapeutic purposes it is also important to understand how OS cells evade Fas-induced cell death triggered by the interaction of Fas with FasL.

Fas-induced apoptosis involves the binding of Fas receptor with its ligand, which induces the recruitment of Fasassociated death domain (FADD) and procaspase-8, leading to the activation of caspase-8 by autocatalytic cleavage. Activated caspase-8 can either directly cleave effector caspases or Bid. Cleaved Bid can then translocate to the mitochondria and induce the activation of capase-9, leading to the activation of effector caspases. ${ }^{9}$ A direct inhibitor of
Fas-mediated apoptosis is cellular FLICE-inhibitory protein (c-FLIP), which functions by competitively binding to FADD and inhibiting the cleavage of procaspase- $-8{ }^{10}$ Cancer cell lines and primary cells and tissues from patients have been found to overexpress C-FLIP. ${ }^{6,24-31}$ As overexpression of c-FLIP is associated with increased resistance to death receptor pathways, several investigators have found that downregulation of c-FLIP results in the sensitization of tumor cell lines to Fas-mediated apoptosis.

Recent studies have shown that upon Fas stimulation, the Fas receptor forms an aggregate and is then redistributed to lipid rafts. ${ }^{11-13}$ It is within these lipid rafts that FADD and caspase-8 are recruited to form the death-inducing signaling complex (DISC) to initiate apoptosis. ${ }^{13}$ It has been reported that Fas signals through ceramide-rich lipid rafts and pretreatment of cells with cholesterol-blocking reagents, which disrupt membrane lipid rafts, inhibits Fas signaling, thereby preventing apoptosis. ${ }^{14}$ Taken together, these studies suggest that Fas redistribution to lipid rafts occurs upon stimulation and is required for Fas-mediated apoptosis.

We previously demonstrated that the histone deacetylase (HDAC) inhibitor MS-275 sensitized Fas ${ }^{-}$OS cells to FasLinduced cell death. ${ }^{15}$ HDAC inhibitors are a promising class of anticancer therapeutics that interfere with the function of HDACs to promote the acetylation of histones, therefore

\footnotetext{
'Department of Pediatrics, The University of Texas MD Anderson Cancer Center, Houston, TX, USA

*Corresponding author: ES Kleinerman, Department of Pediatrics, The University of Texas MD Anderson Cancer Center, 1515 Holcombe Boulevard, Unit 87, Houston, TX 77030, USA. Tel: 713792 8110; Fax; 713794 5042; E-mail: ekleiner@mdanderson.org

Keywords: histone deacetylase inhibitors; Fas; c-FLIP; lipid rafts

Abbreviations: OS, osteosarcoma; c-FLIP, cellular FLICE-inhibitory protein; FADD, Fas-associated death domain; DISC, death-inducing signaling complex; FasL, Fas ligand; sFasL, soluble FasL ligand; $M \beta C D$, methyl- $\beta$-cyclodextrin; shRNA, short hairpin RNA; HDAC, histone deacetylase

Received 06.2.12; revised 25.6.12; accepted 26.6.12; Edited by A Stephanou
} 
affecting gene transcription. Although several groups have identified probable mechanisms of HDAC inhibitor cytotoxicity, the effect of these drugs on the Fas pathway, particularly in OS, is not well understood. Blocking the Fas pathway by introducing dominant-negative FADD abrogated this effect. ${ }^{15}$ This result suggests that the Fas signaling pathway has a role in the ability of MS-275 to sensitize cells to FasL-induced apoptosis. One way to enhance cell sensitivity to FasL is to upregulate the expression of the Fas receptor. Here, we report that MS-275 upregulates the expression of Fas mRNA and protein and induces the redistribution of Fas to membrane lipid rafts. We have also demonstrated that MS-275 decreases c-FLIP expression in OS cells. ${ }^{16}$ In this study, we demonstrate that the downregulation c-FLIP alone also leads to the redistribution of Fas to lipid rafts and sensitizes cells to FasL. Our findings indicate that therapeutic agents that increase Fas localization to lipid rafts or those that decrease c-FLIP expression may be useful in sensitizing OS cells to FasL-induced apoptosis.

\section{Results}

MS-275 sensitizes Fas $^{-}$OS cells to FasL-induced apoptosis. The cytotoxic effects of MS-275 were quantified using human OS cell lines LM7 and CCH-OS-D. Both cell lines express low levels of Fas and are highly metastastic when injected into nude mice. . $^{3,17}$ As expected, these cells were not sensitive to soluble FasL (sFasL) alone (Figure 1). By contrast, treatment of cells with MS-275 increased cell sensitivity to sFasL (Figure 1). Cytotoxic levels in cells treated with both MS-275 and SFasL were higher than those treated with MS-275 alone.

Increased Fas mRNA and protein facilitates FasL binding following treatment of OS cells with MS-275. HDAC inhibitors act on histone acetylation to regulate gene expression. We therefore sought to determine whether MS275 induced a change in Fas expression in LM7 and $\mathrm{CCH}-\mathrm{OS}-\mathrm{D}$ cells. Following treatment of both cell lines with MS-275, there was an increase in Fas mRNA that peaked at $24 \mathrm{~h}$ after addition of MS-275 (Table 1). There was also an increase in Fas protein expression that was time dependent (Figure 2a). In contrast, the cell surface expression of Fas, as detected by flow cytometry, did not change after addition of MS-275 (Figure 2b). However, MS-275 treatment did result in a significant increase in sFasL binding (Figure 2b). These results suggest that the increased Fas protein induced by MS-275 may be in specific membrane compartments not detectable by flow cytometry.

Lipid rafts are required for MS-275-induced sensitization to FasL. Recent studies have demonstrated the importance of membrane lipid raft platforms in Fas receptor signaling. ${ }^{11-13}$ To determine whether there was increased Fas expression in the lipid rafts following MS-275 treatment, we performed sucrose gradient density centrifugation to extract the lipid rafts. The cholesterol content was increased in fractions 3-6, confirming the presence of lipid rafts in these fractions (Figure 3a). Western blot analysis with an antibody against the lipid raft marker, cholera toxin B subunit (CTxB)GM1, confirmed that fractions 3-6 contained lipid raft proteins, whereas fractions 7-9 contained non-raft proteins as determined by transferrin receptor expression, a protein that is excluded from lipid rafts (Figure $3 b$ ). Compared with untreated control cells, cells treated with MS-275 displayed increased Fas expression in the lipid raft fractions (Figure 3b). These results suggest that the increase in Fas protein induced after MS-275 treatment was in lipid raft

Table 1 MS-275 increases Fas mRNA expression

\begin{tabular}{lcc}
\hline & \multicolumn{2}{c}{ Fas mRNA expression (fold increase) } \\
\cline { 2 - 3 } Treatment & LM7 & CCH-OS-D \\
\hline Untreated & 1 & 1 \\
MS-275 12h & $2.3 \pm 0.85$ & $1.9 \pm 0.82$ \\
MS-275 24h & $7.2 \pm 1.49$ & $3.3 \pm 0.76$ \\
MS-275 48h & $3.4 \pm 0.93$ & $1.5 \pm 0.3$ \\
\hline
\end{tabular}

Abbreviation: OS, osteosarcoma. OS cells were treated with $2 \mu \mathrm{M}$ MS-275 for $24 \mathrm{~h}$. RNA was isolated from cells using Trizol reagent, and quantitative real-time PCR was performed using primers specific for Fas. Data show the average and S.D. of five independent experiments.
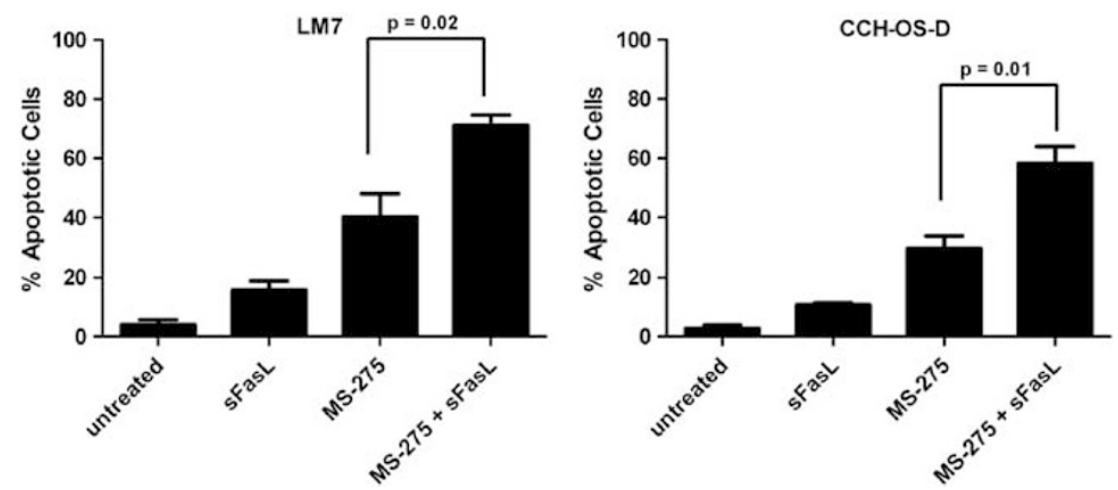

Figure 1 MS-275 sensitizes OS cells to FasL-induced apoptosis. LM7 and CCH-OS-D cells were treated with sFasL for $24 \mathrm{~h}, 2 \mu \mathrm{M}$ MS-275 for $48 \mathrm{~h}$, or both. The percentage of apoptotic cells was determined using the acridine orange/ethidium bromide staining assay. Stained cells were visualized using a fluorescent microscope, and apoptosis was determined as the percentage of apoptotic cells out of 100 counted cells. The average and S.D. of three independent experiments is shown. For MS-275 versus MS-275 and sFasL in combination, $P=0.02$ and $P=0.01$ for LM7 and CCH-OS-D, respectively 


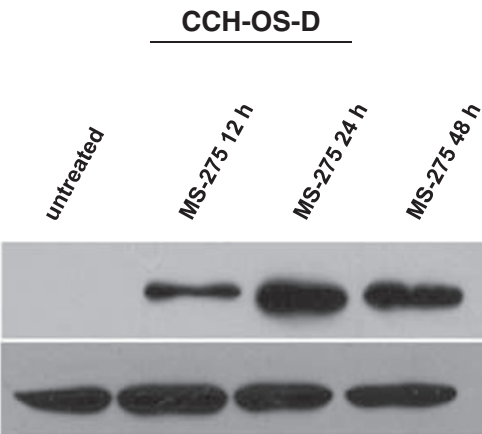

\section{b}

LM7

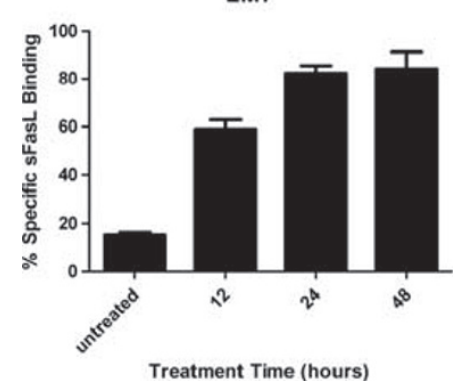

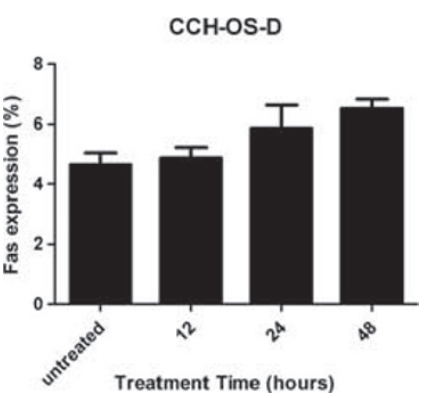

CCH-OS-D

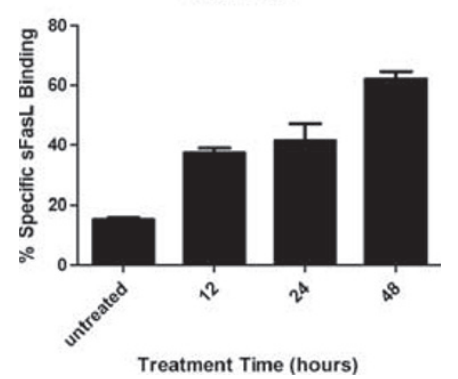

Figure 2 Fas cell surface expression and FasL binding after MS-275 treatment. (a) LM7 and CCH-OS-D cells were treated with MS-275 or control medium, lysed using NP40 buffer, and subjected to western blot analysis using antibodies against Fas and $\beta$-actin. Data represent one of three independent experiments. (b) Cells were treated with $2 \mu \mathrm{M}$ MS-275 for various time periods, then analyzed by flow cytometry using Fas-PE ( $P>0.05$ for untreated versus 12,24 , and $48 \mathrm{~h}$; top panel) or FLAG-PE $(P<0.05$ for untreated versus 12, 24, and $48 \mathrm{~h}$; bottom panel) to detect sFasL binding. A representative example of three independent experiments at $48 \mathrm{~h}$ is shown

microdomains rather than the non-raft regions of the cell surface.

To further confirm the localization of Fas, immunoflouresence staining of Fas and CTxB-GM1 was performed. Untreated cells had low levels of Fas expression localized outside of lipid rafts, whereas cells treated with MS-275 had significantly increased colocalization of Fas within $\mathrm{GM} 1{ }^{+}$lipid rafts (Figures $3 c$ and $d$ ), confirming our results from the fractionation studies.

If localization of Fas in lipid rafts is required for MS-275induced sensitization of cells to sFasL, then inhibiting lipid raft formation should abrogate this effect. To test this hypothesis, the cholesterol sequester methyl- $\beta$-cyclodextrin (M $\beta C D)$ was used to deplete cholesterol and inhibit lipid raft formation in the cells before treatment with MS-275. Reduced cholesterol content was confirmed (Figure 4a). Cells were then treated with MS-275, sFasL, or both and the cytotoxicity was assessed using a clonogenic assay. As anticipated, pretreatment of cells with $\mathrm{M} \beta \mathrm{CD}$ reduced both the cholesterol content and the cytotoxicity associated with the combination of MS-275 and sFasL (Figures $4 a, b$ and c). These results suggest that Fas distribution in the lipid rafts is required for MS-275-induced sensitization to sFasL.

c-FLIP regulates the localization of Fas in lipid rafts. We previously demonstrated that MS-275 downregulates c-FLIP expression in OS cells and in OS lung metastases following oral administration of MS-275. ${ }^{16}$ We therefore investigated whether the MS-275-induced redistribution of Fas to the lipid rafts was mediated by an effect on c-FLIP. Cells with decreased c-FLIP expression were generated by transfection of cells with short hairpin RNA (shRNA) to c-FLIP. Quantitative real-time PCR and western blot analysis confirmed a significant decrease in C-FLIP expression following transfection with shRNA (Figures $5 a$ and b). Fas expression in lipid raft and non-raft fractions was then investigated. Inhibiting C-FLIP increased Fas expression in lipid raft fractions compared with control sh-scrambledtransfected cells (Figure 5c). Amplex red assay was used to confirm separation of lipid raft fractions (Figure $5 \mathrm{~d}$ ). 
a
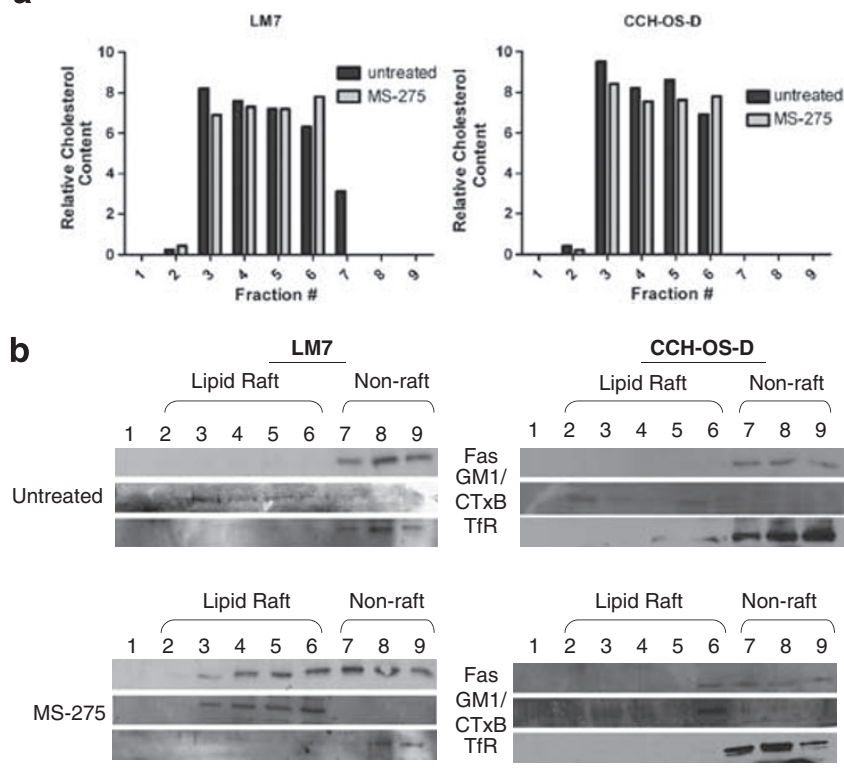

C
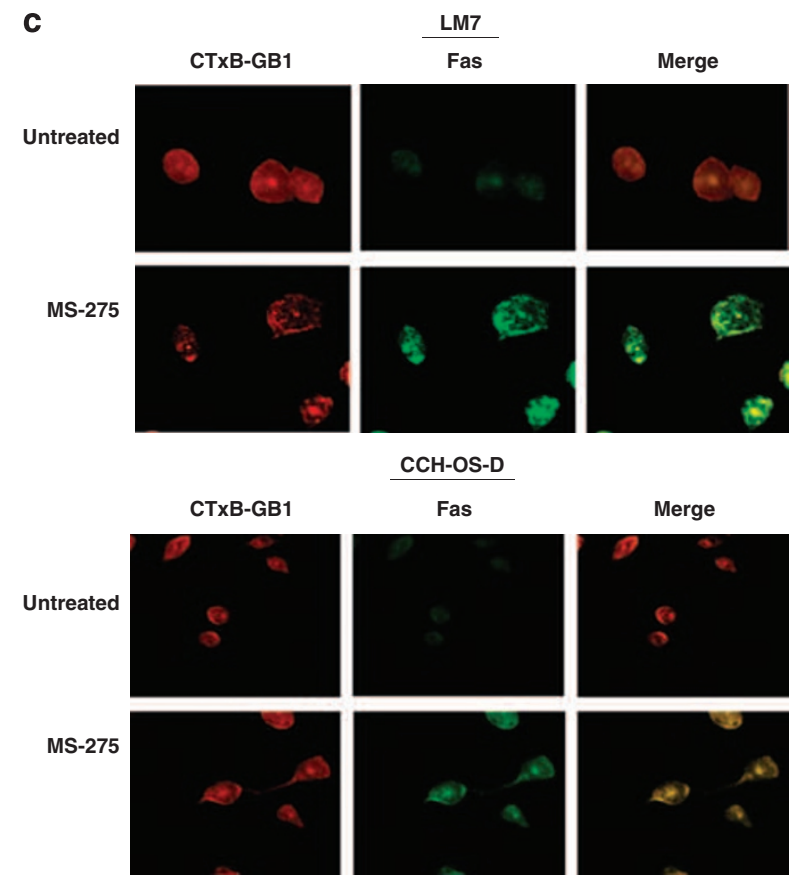

CCH-OS-D
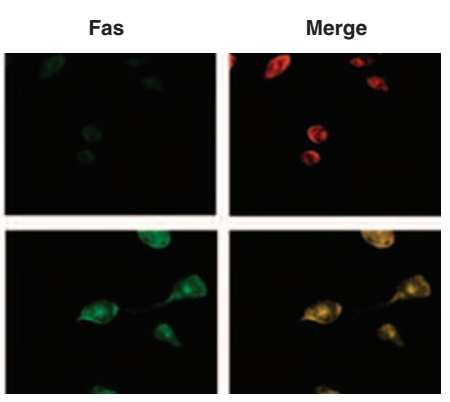

CCH-OS-D
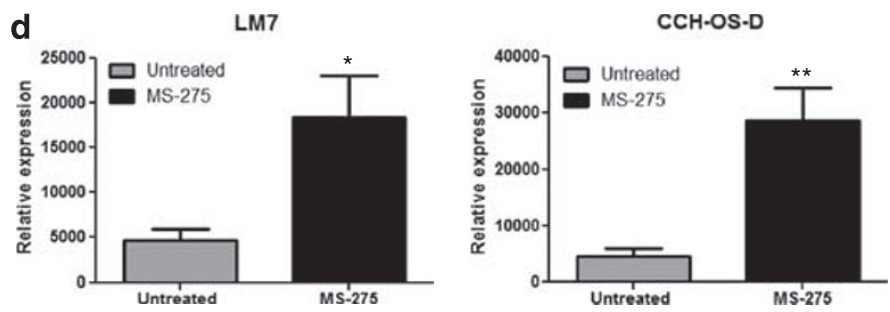

Figure 3 MS-275 increases Fas expression in GM1 + lipid rafts. (a) Amplex red assay was performed to determine the cholesterol content after lipid raft fractionation. Fractions 3-6 were confirmed to contain higher cholesterol content. (b) LM7 and CCH-OS-D cells were treated with $2 \mu \mathrm{M} \mathrm{MS-275}$ or control medium for $48 \mathrm{~h}$. Cells were lysed with Triton X-100, and the lysates were subjected to sucrose density gradient centrifugation. Each gradient fraction was analyzed by western blotting for Fas, CTxB-GM1, and transferrin receptor. Fractions 3-6 were confirmed to contain $\mathrm{GM} 1{ }^{+}$lipid rafts, whereas fractions 7-9 represented proteins found in the non-raft region. (c) Cells were treated with $2 \mu \mathrm{M}$ MS-275 for $48 \mathrm{~h}$, fixed with 4\% paraformaldehyde, and incubated with anti-CTxB-GM1 Alexa Fluor 594 antibody or anti-Fas antibody followed by anti-mouse Alexa Fluor 488 antibody. Stained slides were visualized under a fluorescent microscope. Areas of yellow represent colocalization. All data are representative of three independent experiments. (d) The colocalization of CTXB and Fas-positive areas (yellow area) were quantified by the Simple PCl software. The average relative expression was calculated from the data in five random fields, the bar is the S.E. ${ }^{*} P=0.01$ and ${ }^{* *} P=0.001$ for untreated versus MS-275-treated cells in LM7 and CCH-OS-D, respectively

Further, the inhibition of c-FLIP increased the colocalization of Fas in $\mathrm{GM} 1^{+}$lipid rafts (Figure 5e). These studies indicate that inhibition of c-FLIP can enhance the expression of Fas in lipid raft regions.

\section{Discussion}

Accumulating evidence suggests that downregulation of Fas or corruption of the Fas signaling pathway has a critical role in the formation of OS lung metastases. ${ }^{3,7,18-21}$ In contrast to primary tumors, which contain a mixed population of $\mathrm{Fas}^{+}$ and Fas ${ }^{-}$cells, OS lung metastases are Fas ${ }^{-} .7$ As the lung is one of the few organs to expresses FasL, this implicates the role of the lung microenvironment in the elimination of the $\mathrm{Fas}^{+}$OS cells, leaving only Fas ${ }^{-}$cells to form metastases. Indeed, we have shown that $\mathrm{Fas}^{+}$OS cells are rapidly cleared from the lung while Fas $^{-}$cells remain. ${ }^{19}$ In addition, we previously showed that the upregulation of Fas in established Fas $^{-}$OS lung metastases results in tumor regression. ${ }^{22}$
Therefore, agents that increase Fas expression may have therapeutic potential for patients with OS lung metastases.

Initial studies demonstrated that selective inhibition of HDAC-1 or HDAC-3 resulted in the upregulation of Fas mRNA in OS cells. ${ }^{23,24}$ Here, we showed that MS-275, an HDAC 1/3-specific inhibitor, sensitized Fas ${ }^{-}$OS cells to FasL-induced cell death. We also demonstrated that treatment of cells with MS-275 upregulated Fas mRNA and protein levels. However, flow cytometry was unable to demonstrate an increase in Fas expression on the cell surface. Interestingly, treatment of cells with MS-275 increased binding to sFasL, which suggested an enhanced availability of Fas receptor on the cell membrane and that Fas may be localized to specific membrane compartments.

Much of the recent focus in death receptor signaling has been on the importance of lipid rafts as platforms for Fas receptor signaling. ${ }^{11-14}$ Our results provide the first evidence that treatment of OS cells with an HDAC inhibitor results in increased localization of the Fas receptor in lipid raft 

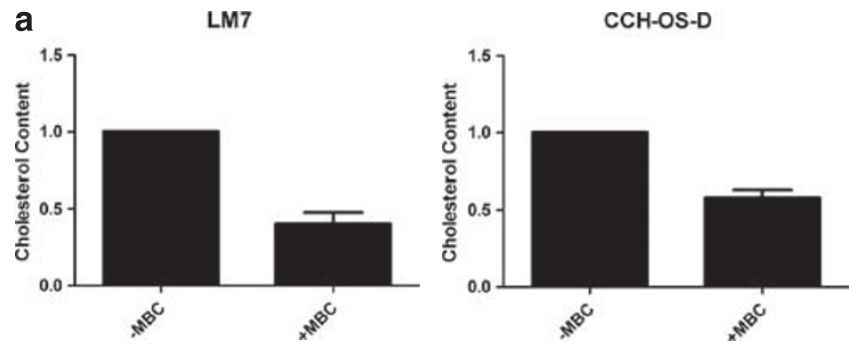

b
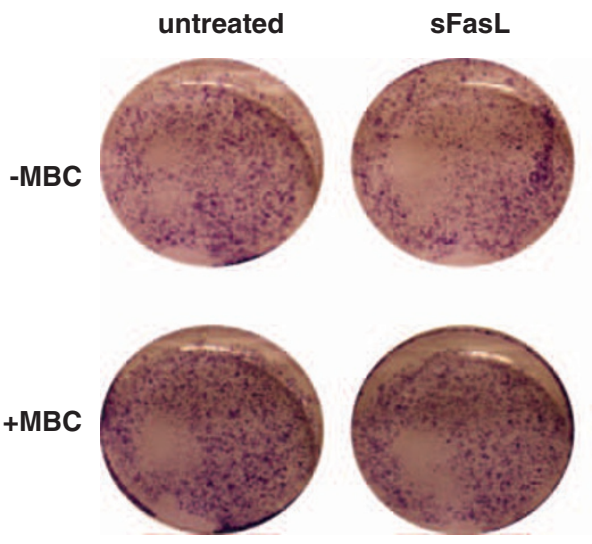

MS-275

MS-275 +sFasL

\section{LM7}
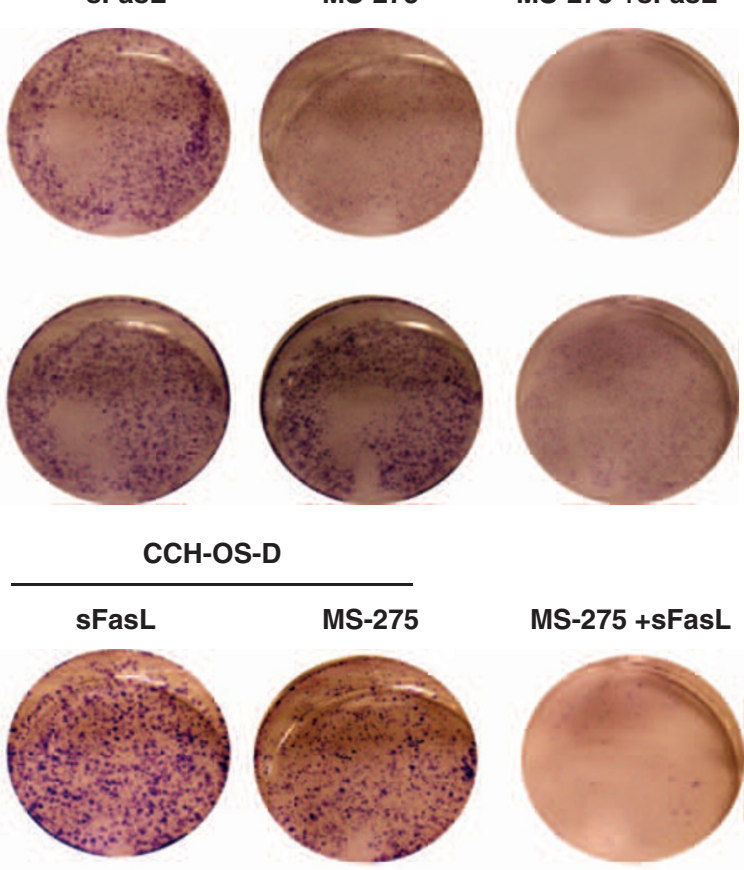

MS-275

MS-275 +sFasL
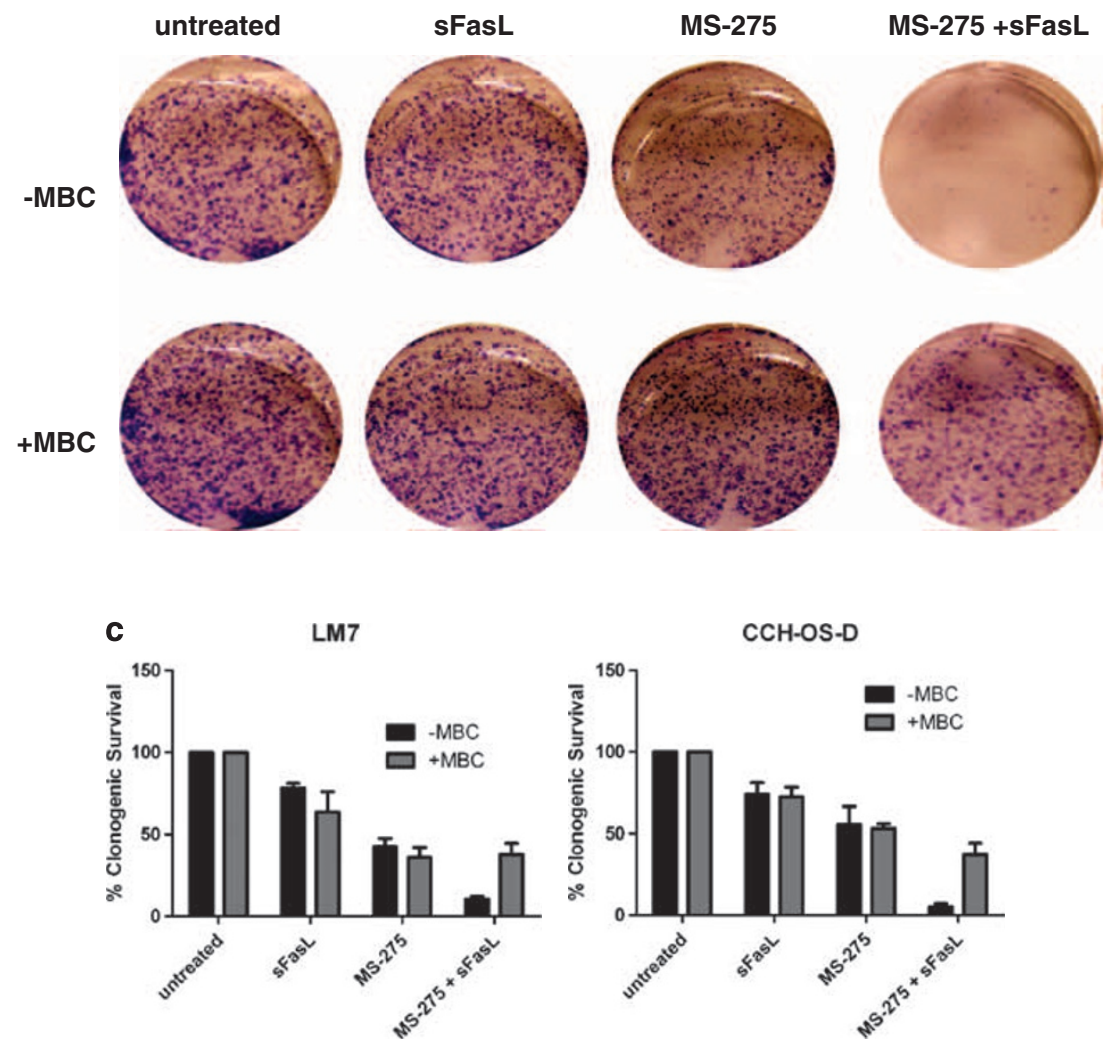

Figure 4 Lipid rafts are required for MS-275-induced sensitization to FasL. LM7 and CCH-OS-D cells were pretreated with $20 \mu \mathrm{M} \mathrm{M} \beta \mathrm{CD}$ for $1 \mathrm{~h}$ and then treated with $2 \mu \mathrm{M}$ MS- 275 for $48 \mathrm{~h}$, sFasL for $24 \mathrm{~h}$, or both. (a) Cholesterol content following M $\beta C D$ treatment was measured using the Amplex red assay. (b) Cytotoxicity with or without M $\beta C D$ was assessed using a clonogenic assay. (c) Quantified number of colonies of four independent experiments. Data show average and S.D. 
a

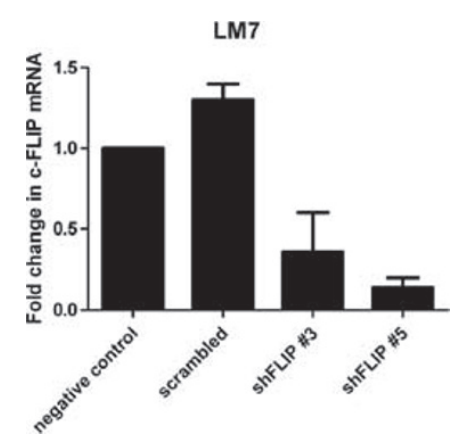

b

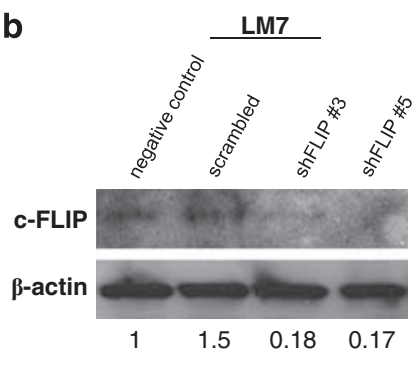

d

c
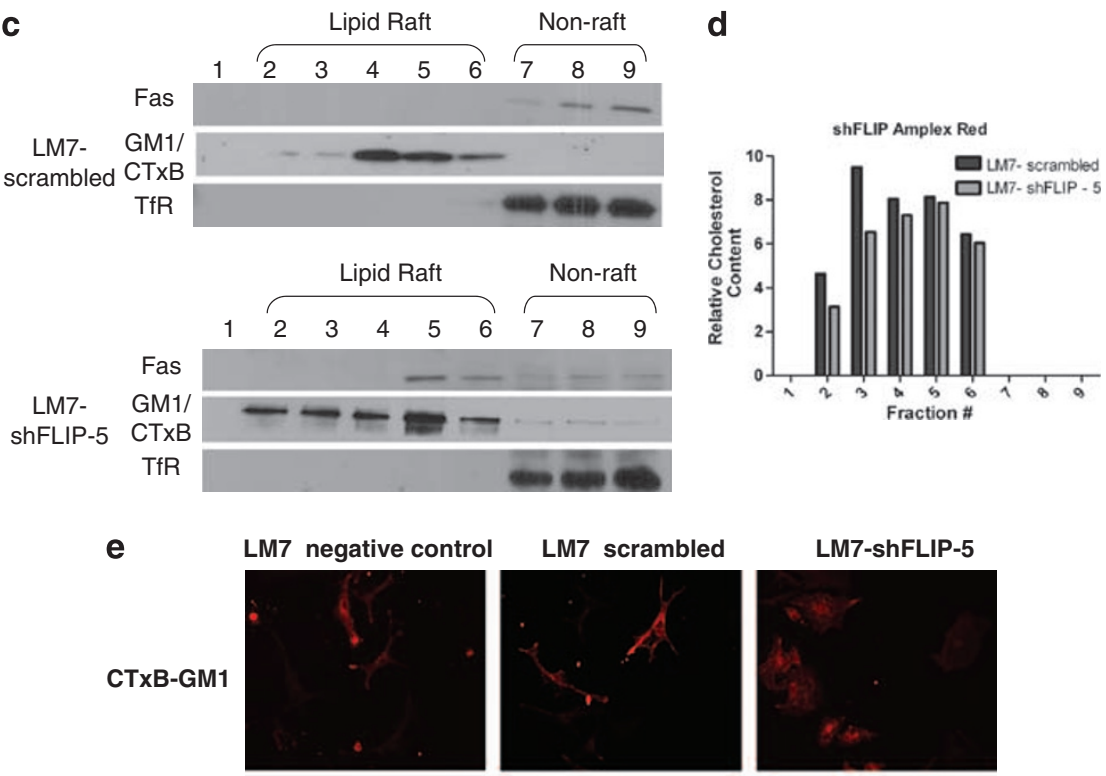

LM7-shFLIP-5
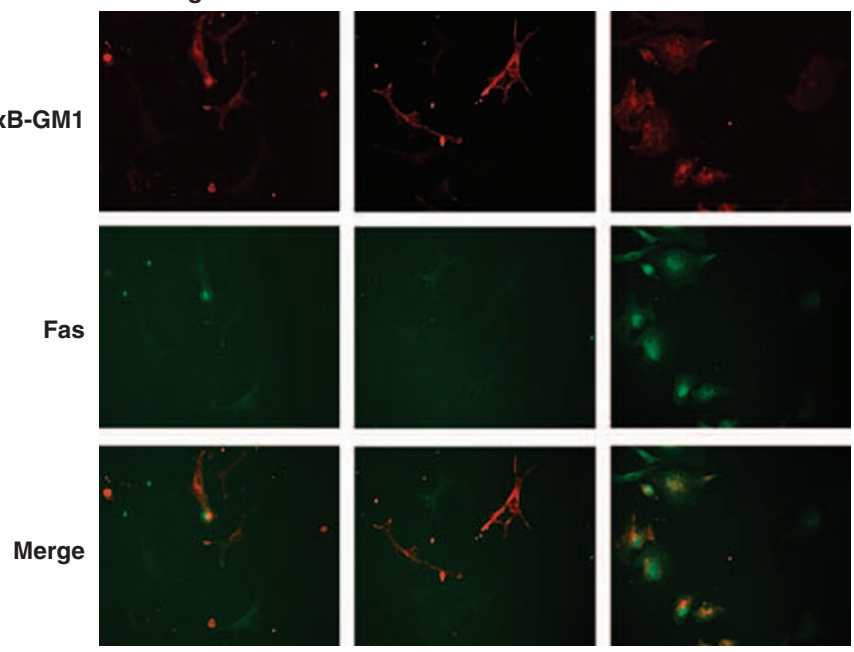

Figure 5 Inhibition of c-FLIP increases localization of Fas in lipid rafts. (a and $\mathbf{b}$ ) Expression of c-FLIP mRNA and protein was determined by quantitative real-time PCR and western blot, respectively, in cells transfected with empty vector (negative control), sh-scrambled clones, and shFLIP-knockdown clones. (c) Cells were lysed with Triton X-100 and lysates subjected to sucrose density gradient centrifugation. Each gradient fraction was analyzed by western blotting for Fas, CTXB-GM1, and transferrin receptor (TfR). (d) Amplex red assay was used to confirm higher cholesterol content in fractions 3-6. Data are representative of three independent experiments. (e) Immunoflourescence staining was performed using anti-CTxB-GM1 Alexa Fluor 594 antibody or anti-Fas antibody followed by anti-mouse Alexa Fluor 488 antibody. Areas of yellow represent colocalization

microdomains, as revealed by our analysis of sucrose gradient fractionation and enhanced colocalization of Fas with $\mathrm{GM} 1^{+}$lipid rafts. These findings are supported by our observation that disruption of lipid rafts with the cholesterol sequester $\mathrm{M} \beta \mathrm{CD}$ abrogated the ability of MS-275 to sensitize OS cells to sFasL.

Death-receptor-induced apoptosis is inhibited by c-FLIP, and overexpression of c-FLIP can confer resistance to Fas-mediated apoptosis. ${ }^{25-29}$ We previously demonstrated that MS-275 treatment results in the downregulation of c-FLIP mRNA and protein in OS cells. ${ }^{16}$ In addition, oral administration of MS-275 in mice with established OS pulmonary metastases decreased c-FLIP protein levels in tumors. ${ }^{16}$ c-FLIP has recently been shown to regulate the distribution of the death receptors DR4 and DR5 in lipid rafts. ${ }^{30}$ We therefore investigated whether altering c-FLIP would affect the localization of Fas in lipid rafts. Using shRNA transfection to inhibit c-FLIP expression, we demonstrated that downregulation of 
c-FLIP in OS cells increased Fas localization in $\mathrm{GM}^{+}$ lipid rafts. Our findings suggest a novel role for c-FLIP in mediating the Fas-specific distribution in lipid rafts as well as the mechanism by which MS-275 increases the localization of Fas to the lipid rafts and sensitizes OS cells to FasL.

There is an ongoing need for novel therapeutic strategies for patients with OS lung metastases. Overall, we demonstrated that the HDAC inhibitor MS-275 sensitizes OS cells to FasL-induced cell death by increasing the expression of Fas in lipid raft microdomains, which correlates with c-FLIP downregulation. Oral MS-275 administered to mice with OS lung metastases resulted in decreased c-FLIP in the tumor nodules and tumor regression. ${ }^{16}$ Thus, modulating Fas distribution in lipid rafts by inhibiting c-FLIP may be a novel therapeutic strategy for treating OS lung metastases cells that have lost Fas expression. Upregulating the Fas expression in the lipid rafts may induce tumor susceptibility to the FasL ${ }^{+}$ lung microenvironment. This is an example of how the tumor microenvironment can be harnessed to eradicate metastatic cells. Therapeutic strategies aimed at altering tumor Fas expression may therefore have a role in the treatment of metastatic OS in the lungs.

\section{Materials and Methods}

Cell lines and reagents. Human OS LM7 and CCH-OS-D cells were maintained in complete Dulbecco's modified Eagle's medium (Whittaker Bioproducts Inc., Walkersville, MD, USA) supplemented with $10 \%$ heat-inactivated bovine serum (Intergen, Purchase, NJ, USA). The LM7 cells were derived from SAOS-2 cells in our laboratory by recycling through the lungs of nude mice. ${ }^{17}$ CCH-OS-D cells were a kind gift from Dennis Hughes, MD, PhD (Division of Pediatrics, The University of Texas MD Anderson Cancer Center). All cell lines were mycoplasma-negative and validated by short tandem repeat DNA Fingerprinting using the AmpFLSTR Identifier kit (Applied Biosystems, Carlsbad, CA, USA). The authenticity of cells was determined by the Characterized Cell Line Core at The University of Texas MD Anderson Cancer Center. Super FasL (human recombinant sFasL) was purchased from Enzo Life Sciences, Inc. (Farmingdale, NY, USA). MS-275 was a generous gift from Syndax Pharmaceuticals, Inc. (Waltham, MA, USA). The lipid raft-inhibiting agent $\mathrm{M} \beta \mathrm{CD}$ was purchased from Sigma-Aldrich, Inc. (St. Louis, MO, USA).

Cytotoxicity and apoptosis assays. Clonogenic assays were performed to assess the cytotoxicity of OS cells after the addition of $10 \mathrm{ng} / \mathrm{ml} \mathrm{sFasL}$ for $24 \mathrm{~h}$, $2 \mu \mathrm{M}$ MS-275 for $48 \mathrm{~h}$, or both. Cells were pretreated with M $\beta C D$ for $1 \mathrm{~h}$. The medium was then removed, and the cells were allowed to grow for 12 days, washed with PBS, and fixed with formalin. Cells were then stained with $0.4 \%$ crystal violet for $30 \mathrm{~min}$, and colonies were counted. Clonogenic assays were performed in triplicate. The acridine orange/ethidium bromide assay was used to measure apoptosis. Cells were plated in 4-well slides and treated with $10 \mathrm{ng} / \mathrm{ml}$ sFasL for $24 \mathrm{~h}, 2 \mu \mathrm{M}$ MS-275 for $48 \mathrm{~h}$, or both. The conventional acridine orange/ ethidium bromide staining protocol was performed following the guidelines from Current Protocols of Immunology. ${ }^{31,32}$ Briefly, an acridine orange/ethidium bromide dye mix, containing $100 \mu \mathrm{g} / \mathrm{ml}$ acridine orange (Sigma-Aldrich, Inc.) and $100 \mu \mathrm{g} / \mathrm{ml}$ ethidium bromide (Sigma-Aldrich, Inc.), was added to each well and the cells were visualized using a fluorescent microscope. Apoptosis was determined as the percentage of apoptotic cells out of all counted cells. The assay was performed in triplicate, and data were averaged.

Flow cytometry and ligand-binding assay. One million OS cells were suspended in FACS buffer (PBS, containing $2 \%$ fetal calf serum and $0.1 \%$ sodium

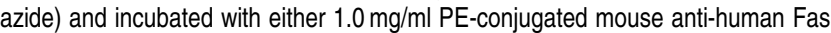
antibody or anti-human FLAG antibody (ProZyme, Hayward, CA, USA) to detect Fas and FasL expression, respectively. PE-conjugated isotype-control IgG antibodies were used as negative controls. Samples were analyzed using a FACScan (Becton Dickinson, Mountain View, CA, USA).
Quantitative real-time PCR. Total RNA was isolated from OS cells using Trizol reagent (Life Technologies, Inc., Gaitherburg, MD, USA). RNA was then reverse transcribed using a Reverse Transcription System (Promega Co., Madison, WI, USA). The resulting CDNA was used for PCR amplification with SYBR green buffer (Bio-Rad Laboratories, Inc., Hercules, CA, USA) and specific primers for Fas (5'-GGCTATAGATCACCTTCATGTA- $3^{\prime}$ and $5^{\prime}$-GCAGTTAACT CAGGGACCAAG- $\left.3^{\prime}\right)$. Primers for $\beta$-actin were used as controls. Quantitative realtime PCR was performed in triplicate using the Bio-Rad 105 real-time PCR detection system. Cycling conditions were $95^{\circ} \mathrm{C}$ for $3 \mathrm{~min}, 45$ cycles of $59^{\circ} \mathrm{C}$ annealing for $30 \mathrm{~s}, 95^{\circ} \mathrm{C}$ for $30 \mathrm{~s}$, and $60^{\circ} \mathrm{C}$ for $1 \mathrm{~min}$.

Western blotting and antibodies. OS cells were collected and lysed using NP-40 buffer (for Fas) or radioimmunoprecipitation buffer (for c-FLIP). Equal amounts of protein were separated on a $10 \%$ SDS-PAGE gel and blotted to the nitrocellulose membrane (Amersham Biosciences Corp., Piscataway, NJ, USA). The membranes were blocked with $5 \%$ non-fat dry milk in PBS containing $0.1 \%$ Tween-20 and then incubated with either anti-Fas antibody (Santa Cruz Biotechnology, Santa Cruz, CA, USA) or anti-FLIP antibody (NF6; Enzo Life Sciences, Inc.) according to the manufacturer's instructions.

Immunoflourescence staining. LM7 and CCH-OS-D cells were cultured in 4-well chamber slides and were either untreated or treated with $2 \mu \mathrm{M} \mathrm{MS}-275$ for $48 \mathrm{~h}$. Cells were fixed with $4 \%$ paraformaldehyde in PBS for $30 \mathrm{~min}$. Samples were then incubated with anti-CTxB antibody conjugated to Alexa Fluor 594 (Molecular Probes, Eugene, OR, USA) at $4^{\circ} \mathrm{C}$ overnight. After washing three times with PBS, the samples were incubated with anti-Fas antibody (BD Biosciences, Franklin Lakes, NJ, USA) at $4{ }^{\circ} \mathrm{C}$ overnight, followed by antimouse Alexa Fluor 488 antibody (Jackson ImmunoResearch, West Grove, PA, USA) at $37^{\circ} \mathrm{C}$ for $2 \mathrm{~h}$. After being washed with PBS, the samples were mounted and visualized with a fluorescent microscope. The colocalization of CTXB- and Fas-positive areas (yellow area) were quantified by the Simple $\mathrm{PCl}$ software (Hamamastu, Bridgewater, NJ, USA) in five random fields. The average relative expression and S.E. was calculated from the data in MS-275-treated and -untreated cells.

Generation of c-FLIP shRNA-expressing clones. To stably knock down c-FLIP expression, OS cells were transduced with retroviruses-expressing shRNA-CFLIP, shRNA-scrambled, or vector pGFP-V-RS (Origene Technologies, Inc., Rockville, MD, USA). Cells were grown in medium containing puromycin to select for stably transfected clones. shRNA constructs were tested for knockdown using western blot analysis with an antibody against c-FLIP (NF6) and by quantitative real-time-PCR using primers against c-FLIP $\left(5^{\prime}\right.$-ACCGAGACTACGAC AGCTTTGTG- $3^{\prime}$ and $5^{\prime}$-CAATGTGAAGATCCAGGAGTGGG-3'). The pGFP-V-RS vector containing the 29-mer shRNA sequence $5^{\prime}$-TGCACAGTTCACCGAGAAGC TGACTTCTT-3' (ID: GI355835) exhibited >80\% c-FLIP knockdown.

Lipid raft fractionation and cholesterol assays. Lipid raft fractions were isolated using the Caveolae/Rafts isolation kit (Sigma-Aldrich, Inc.) performed according to the manufacturer's instructions. Briefly, samples were centrifuged and the pellet was extracted with lysis buffer containing $1 \%$ Triton X-100 and incubated on ice for $30 \mathrm{~min}$. Lysates were mixed with cold OptiPrep density gradient medium and then overlaid with $35,30,25,20$, or $0 \%$ OptiPrep in an ultracentrifuge tube. Samples were then centrifuged at $200000 \times g$ using a fixed angle rotor for $4 \mathrm{~h}$ at $4{ }^{\circ} \mathrm{C}$. Nine 1 -ml fractions were collected from the top to the bottom of each tube. Western blotting was performed with antibodies against Fas (Santa Cruz Biotechnology), HRP-conjugated CTxB (Sigma-Aldrich, Inc.) as a lipid raft marker, or transferrin receptor (Invitrogen Corporation, Carlsbad, CA, USA) as control. An Amplex red assay kit was used to measure the cholesterol content in samples according to the manufacturer's protocol (Invitrogen Corporation).

Statistical analysis. Statistical comparisons of groups were performed using student t-test using the GraphPad Prism 5 software (GraphPad Software, La Jolla, CA, USA) program. A $P$-value of $<0.05$ was deemed as statistically significant.

\section{Conflict of Interest}

The authors declare no conflict of interest. 
Acknowledgements. This work was supported by the National Cancer Institute grant R01 CA42992 (ESK) and the National Institutes of Health core grant CA16672 (ESK), and in part by a fellowship award from the American Legion Auxiliary (KR-B). We thank Dr. Peter Ordentlich and Syndax Pharmaceuticals, Inc. for contributing the MS-275 compound used in our studies. We also acknowledge Dr. Nadezhda V Koshkina for her helpful suggestions. This paper includes data that was presented in a dissertation (by KR-B) to The University of Texas MD Anderson Cancer Center and UT Health Graduate School of Biomedical Sciences: Bindal, Krithi R, 'The histone deacetylase inhibitor, MS-275, sensitizes metastatic osteosarcoma to FasL-induced cell death: a role for c-FLIP' (2012). UT GSGS Dissertations and Theses. Paper 218.

1. Suda $T$, Takahashi $T$, Golstein $P$, Nagata S. Molecular cloning and expression of the Fas ligand, a novel member of the tumor necrosis factor family. Cell 1993; 75: 1169-1178.

2. French LE, Hahne M, Viard I, Radlgruber G, Zanone R, Becker $K$ et al. Fas and Fas ligand in embryos and adult mice: ligand expression in several immune-privileged tissues and coexpression in adult tissues characterized by apoptotic cell turnover. J Cell Biol 1996; 133: 335-343.

3. Worth LL, Lafleur EA, Jia SF, Kleinerman ES. Fas expression inversely correlates with metastatic potential in osteosarcoma cells. Oncol Rep 2002; 9: 823-827.

4. Giuliano AE, Feig S, Eilber FR. Changing metastatic patterns of osteosarcoma. Cancer 1984; 54: 2160-2164.

5. Jia SF, Worth LL, Densmore CL, Xu B, Zhou Z, Kleinerman ES. Eradication of osteosarcoma lung metastases following intranasal interleukin-12 gene therapy using a nonviral polyethylenimine vector. Cancer Gene Ther 2002; 9: 260-266.

6. Gordon N, Kleinerman ES. Aerosol therapy for the treatment of osteosarcoma lung metastases: targeting the Fas/FasL pathway and rationale for the use of gemcitabine. J Aerosol Med Pulm Drug Deliv 2010; 23: 189-196.

7. Gordon N, Koshkina NV, Jia SF, Khanna C, Mendoza A, Worth LL et al. Corruption of the Fas pathway delays the pulmonary clearance of murine osteosarcoma cells, enhances their metastatic potential, and reduces the effect of aerosol gemcitabine. Clin Cancer Res 2007; 13: 4503-4510.

8. Jia SF, Worth LL, Densmore CL, Xu B, Duan X, Kleinerman ES. Aerosol gene therapy with PEI: IL-12 eradicates osteosarcoma lung metastases. Clin Cancer Res, 2003; 9 : $3462-3468$

9. Wajant $\mathrm{H}$. The Fas signaling pathway: more than a paradigm. Science 2002; 296: 1635-1636.

10. Chang DW, Xing Z, Pan Y, Algeciras-Schimnich A, Barnhart BC, Yaish-Ohad S et al. $c$-FLIP(L) is a dual function regulator for caspase-8 activation and CD95-mediated apoptosis. EMBO J 2002; 21: 3704-3714.

11. Grassme H, Jekle A, Riehle A, Schwarz H, Berger J, Sandhoff $K$ et al. CD95 signaling via ceramide-rich membrane rafts. J Biol Chem 2001; 276: 20589-20596.

12. Chakrabandhu K, Herincs Z, Huault S, Dost B, Peng L, Conchonaud F et al. Palmitoylation is required for efficient Fas cell death signaling. EMBO J 2007; 26: 209-220.

13. Chaigne-Delalande B, Moreau JF, Legembre P. Rewinding the DISC. Arch Immunol Ther Exp 2008; 56: 9-14.

14. Bionda C, Athias A, Poncet D, Alphonse G, Guezguez A, Gambert P et al. Differential regulation of cell death in head and neck cell carcinoma through alteration of cholesterol levels in lipid rafts microdomains. Biochem Pharmacol 2008; 75: 761-772.

15. Koshkina NV, Rao-Bindal K, Kleinerman ES. Effect of the histone deacetylase inhibitor SNDX-275 on fas signaling in osteosarcoma cells and the feasibility of its topical application for the treatment of osteosarcoma lung metastases. Cancer 2011; 117: 3457-3467.

16. Rao-Bindal K, Kleinerman ES. The histone deacetylase inhibitor, MS-275, sensitizes osteosarcoma cells and osteosarcoma lung metastases to FasL-induced cell death by the downregulation C-FLIP. Proceedings of the 102nd Annual Meeting of the American Association for Cancer Research, Orlando Florida: Philadelphia (PA): AACR 2011 April 2-5.

17. Jia SF, Worth LL, Kleinerman ES. A nude mouse model of human osteosarcoma lung metastases for evaluating new therapeutic strategies. Clin Exp Metastasis 1999; 17: 501-506.

18. Gordon N, Kleinerman ES. The role of Fas/FasL in the metastatic potential of osteosarcoma and targeting this pathway for the treatment of osteosarcoma lung metastases. Cancer Treat Res 2009; 152: 497-508.

19. Koshkina NV, Khanna C, Mendoza A, Guan H, DeLauter L, Kleinerman ES. Fas-negative osteosarcoma tumor cells are selected during metastasis to the lungs: the role of the Fas pathway in the metastatic process of osteosarcoma. Mol Cancer Res 2007; 5 : 991-999.

20. Gordon N, Arndt CA, Hawkins DS, Doherty DK, Inwards CY, Munsell MF et al. Fas expression in lung metastasis from osteosarcoma patients. J Pediatr Hematol Oncol 2005; 27: 611-615.

21. Lafleur EA, Koshkina NV, Stewart J, Jia SF, Worth LL, Duan X et al. Increased Fas expression reduces the metastatic potential of human osteosarcoma cells. Clin Cancer Res 2004; 10: 8114-8119.

22. Lafleur EA, Jia SF, Worth LL, Zhou Z, Owen-Schaub LB, Kleinerman ES. Interleukin (IL)-12 and IL-12 gene transfer up-regulate Fas expression in human osteosarcoma and breast cancer cells. Cancer Res 2001; 61: 4066-4071.

23. Escaffit $F$, Vaute $O$, Chevillard-Briet M, Segui B, Takami Y, Nakayama T et al. Cleavage and cytoplasmic relocalization of histone deacetylase 3 are important for apoptosis progression. Mol Cell Biol 2007; 27: 554-567.

24. Senese S, Zaragoza K, Minardi S, Muradore I, Ronzoni S, Passafaro A et al. Role for histone deacetylase 1 in human tumor cell proliferation. Mol Cell Biol 2007; 27: 4784-4795.

25. Scaffidi C, Schmitz I, Krammer PH, Peter ME. The role of C-FLIP in modulation of CD95induced apoptosis. J Biol Chem 1999; 274: 1541-1548.

26. Irmler M, Thome M, Hahne M, Schneider P, Hofmann K, Steiner V et al. Inhibition of death receptor signals by cellular FLIP. Nature 1997; 388: 190-195.

27. Lavrik IN. Regulation of death receptor-induced apoptosis induced via CD95/FAS and other death receptors. Mol Biol 2011; 45: 173-179.

28. Beneteau M, Daburon S, Moreau JF, Taupin JL, Legembre P. Dominant-negative Fas mutation is reversed by down-expression of C-FLIP. Cancer Res 2007; 67: 108-115.

29. Bagnoli M, Canevari S, Mezzanzanica D. Cellular FLICE-inhibitory protein (c-FLIP) signalling: a key regulator of receptor-mediated apoptosis in physiologic context and in cancer. Int J Biochem Cell Biol 2010; 42: 210-213.

30. Song JH, Tse MC, Bellail A, Phuphanich S, Khuri F, Kneteman NM et al. Lipid rafts and nonrafts mediate tumor necrosis factor related apoptosis-inducing ligand induced apoptotic and nonapoptotic signals in non small cell lung carcinoma cells. Cancer Res 2007; 67: 6946-6955.

31. Coligan JEKA, Margulies DH, Shevach EM, Strober W. Current Protocols in Immunology. In: Coico R: John Wiley \& Sons I (ed). Related Isolation Procedures and Functional Assays, vol. 1, 1995.

32. Ribble $D$, Goldstein NB, Norris DA, Shellman YG. A simple technique for quantifying apoptosis in 96-well plates. BMC Biotechnol 2005; 5: 12.

Cell Death and Disease is an open-access journal published by Nature Publishing Group. This work is licensed under the Creative Commons Attribution-NonCommercial-No Derivative Works 3.0 Unported License. To view a copy of this license, visit http://creativecommons.org/licenses/by-nc-nd/3.0/ 